\title{
FRACTIONAL MODELS IN SPACE FOR DIFFUSIVE PROCESSES IN HETEROGENEOUS MEDIA WITH APPLICATIONS IN CELL MOTILITY AND ELECTRICAL SIGNAL PROPAGATION
}

\author{
NICOLE CUSIMANO
}

(Received 11 November 2015; first published online 10 February 2016)

2010 Mathematics subject classification: primary 35R11; secondary 26A33, 65N06, 65N25, 65N35, $92 \mathrm{~B} 05$.

Keywords and phrases: fractional Laplacian, insulated bounded domain, spectral methods, mathematical biology, cardiac electrophysiology.

Fractional calculus, that is, calculus involving derivatives and integrals of noninteger order, was considered for many years from a purely abstract mathematical perspective. However, in the last few decades, fractional operators have been used as modelling tools in a variety of practical disciplines (such as physics, engineering, chemistry, rheology and economics) due to their success in reproducing solution behaviours that significantly deviate from that typically obtained with standard models, as in the case of anomalous diffusion. The interest in fractional operators linked to practical applications is increasingly growing; however, there are still fundamental issues and implementation aspects that have to be addressed for a rigorous implementation of these operators in a wider variety of problems. Important considerations involve for example the restriction of the nonlocal definition of the operators to bounded domains and the role played by the boundary conditions.

In this thesis we focus our attention on reflecting boundary conditions, essentially extending the concept of standard homogeneous Neumann boundary conditions to nonlocal problems. We derive a discrete representation of the space-fractional operator

Thesis submitted to Queensland University of Technology in May 2015; degree approved on 12 May 2015; principal supervisor Kevin Burrage, associate supervisor Pamela Burrage.

This is an Open Access article, distributed under the terms of the Creative Commons Attribution licence (http://creativecommons.org/licenses/by/4.0/), which permits unrestricted re-use, distribution, and reproduction in any medium, provided the original work is properly cited.

(c) 2016 Australian Mathematical Publishing Association Inc. 0004-9727/2016 \$16.00 
embedding reflecting boundary conditions on a finite one-dimensional domain and formulate a theorem providing the analytic expression of its spectrum. We then compare this operator with the one obtained with the matrix transfer technique when standard homogenous Neumann boundary conditions are considered, and show the equivalence of their spectrum in the limit as the number of discretisation points approaches infinity.

Justified by the above theoretical considerations, we adopt the spectral definition of the fractional Laplacian on insulated bounded domains in order to obtain a spacefractional modification of some models of interest for two particular applications (one in biology and one in electrophysiology). We then use the spectral decomposition method to compute their solution in both the standard and fractional cases, and analyse how the fractional component affects the solution of these models.

In particular, in the space-fractional modification of the Fisher-Kolmogoroff model for cell migration on an insulated bounded domain we see that solutions evolving as travelling waves in the standard case no longer advance with constant shape and speed in the fractional case. In fact, we observe an acceleration of the solution profile towards the stable steady state of the system that translates mathematically into the exponential advancing of the level sets of the fractional solution. Inspired by in vivo experimental recordings of the speed of advancing neural crest-derived cells in the gut of mice embryos, we then propose a variable-order fractional modification of the model with the aim of characterising the migration pattern observed in different regions of the domain with different values of the fractional order.

Finally, we consider the space-fractional modification of two examples of excitable media models, namely the Fitzhugh-Nagumo and the Beeler-Reuter monodomain models. In one spatial dimension, the same electrical stimulus applied to the system in the standard case and generating a travelling pulse moving through the domain away from the stimulus site in the fractional case still produces a travelling pulse with shape dictated by the particular cell model used. However, as the fractional order decreases, we observe a reduction in the conduction velocity (and hence a later depolarisation of any given point in space), a slight decrease in the action potential peak (for the Beeler-Reuter model also a slightly more pronounced early depolarisation phase) and increasing dispersion of the action potential duration as the travelling pulse moves away from the stimulus site. In two spatial dimensions, we study two different operators introducing nonlocality on an insulated rectangular domain: the sum of two one-dimensional fractional Laplacians with reflecting boundary conditions in each spatial dimension (allowing us to consider also different fractional orders in the two spatial directions) and a spectral definition for the two-dimensional fractional Laplacian as a whole. As the level of nonlocality in the problem increases (and the fractional orders of the two operators decrease), the same type of qualitative changes found in the one-dimensional case is seen here in the solution. However, we observe differences in the curvature of the travelling pulse front between the two options. 
Furthermore, if different fractional orders are used for the first option in different directions, even under the assumption of isotropic conductivity values, the temporal evolution of the two-dimensional travelling pulse is not symmetric with respect to the two directions and a faster advancing is observed along the direction characterised by the higher fractional order.

\section{NICOLE CUSIMANO,}

ARC Centre of Excellence for Mathematical and Statistical Frontiers, Queensland University of Technology, Brisbane, 4000 QLD, Australia e-mail: nicole.cusimano@outlook.com 